\title{
I.j) Anesthesia / Early recovery / Preoperative preparation free papers
}

\section{CO35}

RENAL PROTECTION WITH SODIUM THIOSULFATE DURING HYPERTHERMIC INTRAPERITONEAL CHEMOTHERAPY (HIPEC) WITH CISPLATIN

\author{
N. Laplace ${ }^{1}$, V. Kepenekian ${ }^{1}$, F. Mercier ${ }^{1}$, A. Friggeri' ${ }^{2}$, N. Bakrin ${ }^{1}$, G. Passot ${ }^{1}$, D. Vaudoyer ${ }^{1}$, O. Glehen ${ }^{1}$ \\ ${ }^{1}$ Department of Surgical Oncology, Centre Hospitalier Lyon Sud - Pierre Bénite (France), ${ }^{2}$ Department \\ of Anaesthesiology, Centre Hospitalier Lyon Sud - Pierre Bénite (France)
}

\section{Objectives}

Cytoreductive surgery combined with hyperthermic intraperitoneal chemotherapy (HIPEC) has shown to provide benefits in the management of peritoneal carcinomatosis. Cisplatin (CDDP) is one of the most frequently used drugs for peritoneal perfusion. A major restriction is that CDDP causes renal toxicity and acute renal failure sometime leading to chronic renal failure needing renal replacement therapy. The aim of our study was to assess the impact of sodium thiosulfate in preventing renal impairment (RI) during CDDP HIPEC.

\section{Methods}

This prospective study assessed the RI rates for all patients which underwent a CDDP HIPEC between two successive periods in our department: without introduction of sodium thiosulfate (Period nST: November 2016 to September 2017) and sodium thiosulfate utilization period (Period ST: October 2017 to March 2018). During the Period ST, patients were treated with sodium thiosulfate perfusion at $9 \mathrm{mg} / \mathrm{m}^{2}$ prior to HIPEC. The $\mathrm{RI}$ was defined by postoperative creatinemia $>1.6$ (WHO grade I toxicity). The impact of sodium thiosulfate treatment was evaluated by comparison of the RI rates between the two periods.

\section{Results}

During Period ST, 0 of 31 patients $(0 \%)$ developed RI versus 11 of 35 patients $(31,43 \%)$ during Period nST $(p<0.05)$. Two of them required definitive hemodialysis. Baseline characteristics, background circumstances, indications and laboratory parameters before HIPEC were comparable between the two groups, as well as CDDP dose use during HIPEC.

\section{Conclusion}

None of the 31 patients treated with sodium thiosulfate $9 \mathrm{mg} / \mathrm{m}^{2}$ developed renal impairment. Sodium thiosulfate appears to be an effective drug for preventing renal toxicity from the toxic effects of CDDP used for HIPEC in patients with peritoneal carcinomatosis. 
CO36

A RANDOMISED CONTROLLED TRIAL TO COMPARE ADMINISTRATION OF PROTHROMBIN COMPLEX CONCENTRATE WITH STANDARD HAEMOSTASIS MANAGEMENT IN ADULT PATIENTS UNDERGOING CYTOREDUCTIVE SURGERY AND HYPERTHERMIC INTRAPERITONEAL CHEMOTHERAPY (CRS/HIPEC) REQUIRING MASSIVE TRANSFUSION

\author{
M.S. Alshahrani ${ }^{1}$, O.M. Fisher ${ }^{1}$, S.J. Ho ${ }^{2}$, N.A. Alzahrani ${ }^{1}$, W. Liauw ${ }^{2}$, D.L. Morris ${ }^{1}$ \\ ${ }^{1}$ Liver and Peritonectomy Unit, St. George Hospital - Sydney (Australia), ${ }^{2}$ Cancer Care Center, St. George \\ Hospital - Sydney (Australia)

\section{Objectives}

To determine whether PCC (Prothrombinex-VF) can safely and effectively be given in patients undergoing cytoreductive surgery and hyperthermic intraperitoneal chemotherapy (CRS/HIPEC).

\title{
Methods
}

All patients planned for CRS/HIPEC at St George Hospital who were judged to be at high risk of massive blood transfusion were randomized to receiving Prohthrombinex vs standard treatment. Intra-operative and post-operative clinical data were collected and compared.

\section{Results}

80 patients were randomized into the two groups between 2013-2017, with 40 patients in each arm. There were no significant differences in baseline demographic characteristics. There were no significant differences between groups in terms of peritoneal carcinomatosis index $(\mathrm{PCl})$, duration of surgery, ICU stay or total hospital stay. Patients in the PCC group had lower incidence of hemorrhage with surgery (defined as requirements of more than 2 units of PRBC, $p=0.045$ ). Intra-operatively, this group required less PRBC transfusion (7.08 vs $4.70, p=0.07)$, less FFP (7.98 vs $2.23, p<0.001)$, less platelets $(0.7$ vs 0.33 , $p=0.043)$, and less cryoprecipitate (9.38 vs $7.40, p=0.282)$. There were no significant differences in terms of post-operative complications, with an observation of less thromboembolic events in the PCC group (15\% vs $27.5 \%, p=0.17)$.

\section{Conclusion}

Use of Prothrombinex in patients undergoing CRS/HIPEC resulted in less blood transfusion. Despite previous reports of high thromboembolic events, our data does not support that. 


\title{
I.j) Anesthesia / Early recovery / Preoperative preparation free papers
}

CO37

HYPERTHERMIC INTARPERITONEAL CHEMOTHERAPY ENCHANCES BLOOD COAGULATION

\author{
M. Tuovila, T. Erkinaro, H. Takala, E.R. Savolainen, P. Laurila, P. Ohtonen \\ Oulu University Hospital - Oulu (Finland)
}

Objectives

Despite mechanical and pharmacological prophylaxis, postoperative thromboembolism remains a significant cause of prolonged recovery for patients undergoing cytoreductive surgery (CRS) and hyperthermic intraperitoneal chemotherapy (HIPEC). Most of these thrombotic complications occur after hospital discharge. Thromboelastography (TEG) has been demonstrated to be able to detect hypercoagulable states and predict thromboembolic complications after surgery. In this study we assessed the impact of CRS and HIPEC on TEG values up to 3 months after the procedure.

\section{Methods}

We studied venous blood samples from 15 patients undergoing CRS and HIPEC in Oulu University Hospital during 2015-2017. All patients received enoxaparine $40 \mathrm{mg} \times 2$ for thrombosis prophylaxis. TEG parameters reaction time $(R)$, kinetics time $(K)$, angle (alpha), maximum amplitude (MA) and lysis percent at 60 min (LY60) were determined preoperatively, on the first postoperative day, at hospital discharge and 3 months after the procedure. We also determined platelets, thromboplastin time (P-TT), fibrinogen and D-dimer at each time point. All the patients were screened for deep venous thrombosis (DVT) by Duplex ultrasound preoperatively and at hospital discharge. The occurrence of DVTs and pulmonary embolism (PE) between discharge and 3 months control were recorded.

\section{Results}

Preoperatively, the median MA was elevated $(69.6 \mathrm{~mm})$ reflecting increased clot strength. Other TEG parameters as well as conventional coagulation tests were within normal limits. During the hospital stay there was a significant increase in the mean alpha (68.5 vs $\left.73.2^{\circ}, p=0.002\right)$ and MA (69.6 vs $77.8 \mathrm{~mm}$, $p<0.0001)$. There was also a decrease in the mean P-TT (92 vs 79\%, p =0.02) and an increase in the mean platelets $(282$ vs $459 \times 109, p<0.05)$, fibrinogen $(3.7$ vs $6.2 \mathrm{~g} / \mathrm{l}, \mathrm{p}<0.0001)$ and $\mathrm{D}$-dimer $(1.2$ vs $8.0 \mathrm{mg} / \mathrm{l}, \mathrm{p}<0.0001)$. After hospital discharge both TEG parameters and the conventional coagulation tests returned to normal limits. No DVTs were found during the study period but one patient had a PE after hospital discharge.

\section{Conclusion}

CRS with HIPEC causes a hypercoagulative state which can be detected by TEG and conventional coagulation tests. All changes in blood coagulation normalize within 3 months after the operation. 